\title{
Correction to: Comparative Evaluation of Various Feature Weighting Methods on Movie Reviews
}

\author{
S. Sivakumar and R. Rajalakshmi
}

\section{Correction to:}

Chapter "Comparative Evaluation of Various Feature Weighting Methods on Movie Reviews" in:

H. S. Behera et al. (eds.), Computational Intelligence in Data Mining, Advances in Intelligent Systems and Computing 711, https://doi.org/10.1007/978-981-10-8055-5_64

In the original version of the book, the following post-publication corrections have been incorporated in the chapter "Comparative Evaluation of Various Feature Weighting Methods on Movie Reviews":

The second author, R. Rajalakshmi, has been changed as the corresponding author.

The second affiliation "Department of Computer Science, Dhanalakshmi College of Engineering, Chennai 600127, Tamil Nadu, India" of the corresponding author, R. Rajalakshmi, has been removed.

The correction chapter and the book have been updated with the changes.

The updated version of this chapter can be found at https://doi.org/10.1007/978-981-10-8055-5_64 